\title{
The Faraday Isolator, Detailed Balance and the Second Law
}

\author{
George S. Levy \\ Entropic Power, 3980 Del Mar Meadows, San Diego, CA, USA \\ Email: glevy@entropicpower.com
}

How to cite this paper: Levy, G.S. (2017) The Faraday Isolator, Detailed Balance and the Second Law. Journal of Applied Mathematics and Physics, 5, 889-899. https://doi.org/10.4236/jamp.2017.54078

Received: March 27, 2017

Accepted: April 27, 2017

Published: April 30, 2017

Copyright $\odot 2017$ by author and Scientific Research Publishing Inc. This work is licensed under the Creative Commons Attribution International License (CC BY 4.0).

http://creativecommons.org/licenses/by/4.0/

\section{(c) (i) Open Access}

\begin{abstract}
A Faraday isolator is shown to develop a temperature difference between its input and output, but still complies with the second law when all the heat carriers, in this case, photons are homogeneous and indistinguishable. This result is a consequence of the H-theorem which assumes homogeneity and indistinguishability of particles. However, when a thermal feedback path is added, in which heat carriers have physical properties different from the photons in the isolator, then a heterogeneous system is formed not covered by the $\mathrm{H}$-theorem, and the second law is violated.
\end{abstract}

\section{Keywords}

Faraday Isolator, Detailed Balance, Second Law, Non-Maxwellian, Entropy, H-Theorem, Statistical Mechanics, Perpetual Motion Machine, Statistical Symmetry, Indistinguishability

\section{Introduction}

Reciprocity in absorption and emission is a requirement of detailed balance and expressed by Kirchhoff law of radiation for any wavelength and for any direction.

$$
\alpha(\omega, \theta, \varphi)=\varepsilon(\omega, \theta, \varphi)
$$

In other words, the absorptivity $\alpha$ is equal to the emissivity $\varepsilon$ for any value of frequency $\omega$ and polar coordinate angles $\theta$ and $\varphi$. This law is conventionally accepted, yet non-reciprocity of transmission and reflection has been the puzzlement [1] to scientists as it appears to violate the principle of detailed balance and the second law. Non-reciprocal devices are used in a multitude of applications, for example Faraday isolators and optical and microwave circulators. This paper discusses how such non-reciprocity leads to the breakdown of detailed balance and the second law.

The Faraday isolator is a non-reciprocal optical device, i.e., a light diode. It 
comprises an input linear polarizer, a polarization rotator and an output linear polarizer at $45^{\circ}$ from the input polarizer. Light entering the isolator through the input is linearly polarized, then rotated by a $45^{\circ}$ angle and exits unimpeded through the output polarizer. Light entering through the output is polarized, and then rotated another $45^{\circ}$ thereby, encountering the first polarizer at a $90^{\circ}$ angle and being stopped. The unidirectionality of the device seems to indicate that objects downstream of the light flow should get warmer and those upstream should get colder.

Wien [2] attempts to prove that Faraday isolators cannot violate the second law. He describes a thought experiment involving two black bodies A and B separated by a Faraday isolator comprised of polarizers $\mathrm{X}$ and $\mathrm{Y}$ and a Faraday rotator R. The polarizers are nicol prisms which, he assumes, transmit half of the light and reflect the other half. Wien's analysis assumes that the black bodies are initially at the same temperature. The analysis is illustrated in Figure 1 in which the width of the channels corresponds to the magnitude of heat flow.

Half of the light coming from $\mathrm{A}$ is reflected back to A by the nicol $\mathrm{X}$. The other half undergoes a $45^{\circ}$ rotation, traverses nicol $\mathrm{Y}$ and reaches black body $\mathrm{B}$.

Half of the light coming from B is reflected back to B by nicol Y. The second half is rotated by $45^{\circ}$ and as it reaches nicol X, is totally reflected toward B. Wien then carelessly assumes that this polarized light crosses nicol $\mathrm{Y}$ and reaches $\mathrm{B}$. $\mathrm{He}$ concludes that $\mathrm{B}$ receives three times more energy than A.

Wien's careless assumption is erroneous. This error is corrected by Rayleigh as described further below. In any case, it is instructive to continue with Wien's reasoning, and then discuss Rayleigh's correction.

To avoid a conflict with the second law, Wien proposes two solutions (original quote in French) [2]:

"On peut résoudre de deux façons différentes cette contradiction avec le second principe de la Thermodynamique.

Ou bien le magnétisme de la substance douée du pouvoir rotatoire est détruit par la radiation qui la traverse, c'est à dire que si la rotation est produite par des aimants permanents ces aimants sont affaiblis.

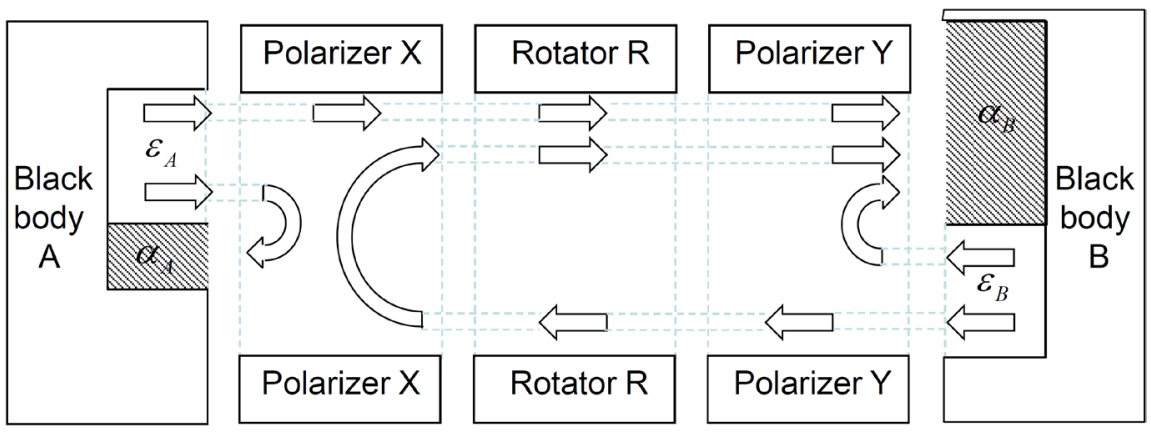

Figure 1. Wien's thought experiment using nicol prisms which transmit half of the light and reflect the other half. According to Wien, black body B receives three times more light than black body A. The figure shows the black bodies in an initial non-equilibrium state. 
Ou tous les corps transparents doués du pouvoir rotatoire absorbent la lumière en faisant tourner son plan de polarization."

Translated to English, Wien proposes that, either light that goes through the rotator attenuates the magnetic field, or that the rotator absorbs light as a function of the rotation imparted to light.

Neither of his proposed solutions is acceptable. His first argument requires light to weaken the magnetic field, thereby impacting the bi-directional operation of the rotator. The complete elimination of the rotator's operation is obviously not possible because, then, Faraday isolators would not work. The partial reduction of the rotator's operation can easily be countered by increasing the length of the rotator to restore its function.

His second argument requires light to be asymmetrically absorbed by the rotator as a function of the orientations of the non-local polarizers. In other words, light from one of the polarizers would have to be preferentially absorbed compared to light from the other polarizer. There is no known physical mechanism by which this effect can be achieved.

Rayleigh [3] properly completes Wien's thought experiment, thereby correcting Wien's omission. As shown in Figure 2 Rayleigh finds that, after the reflection from $\mathrm{X}$, light acquires another $45^{\circ}$ rotation as it traverses the rotator a third time and instead of crossing nicol $\mathrm{Y}$ and reaching $\mathrm{B}$, light is reflected by nicol $\mathrm{Y}$ towards A.

At that point, the light has the correct polarization to cross nicol $\mathrm{X}$ and reach A. Surprisingly, Raleigh shows that the isolator is not unidirectional and he concludes that the two black bodies receive the same amount of light and the second law is not violated.

Rayleigh's argument leaves one to wonder how an isolator can function properly if internal reflections cancel its unidirectional operation even under normal operation as a light diode. Both Wien and Rayleigh's arguments are faulty as they rely on a non-working isolator design. In actual nicol-based isolators, light is not reflected backward by the nicols but deflected and absorbed by surrounding materials and collimators.

Mungan [1] asserts without a detailed proof that no violation of the second law occurs because heat from the hot object is absorbed by the isolator and

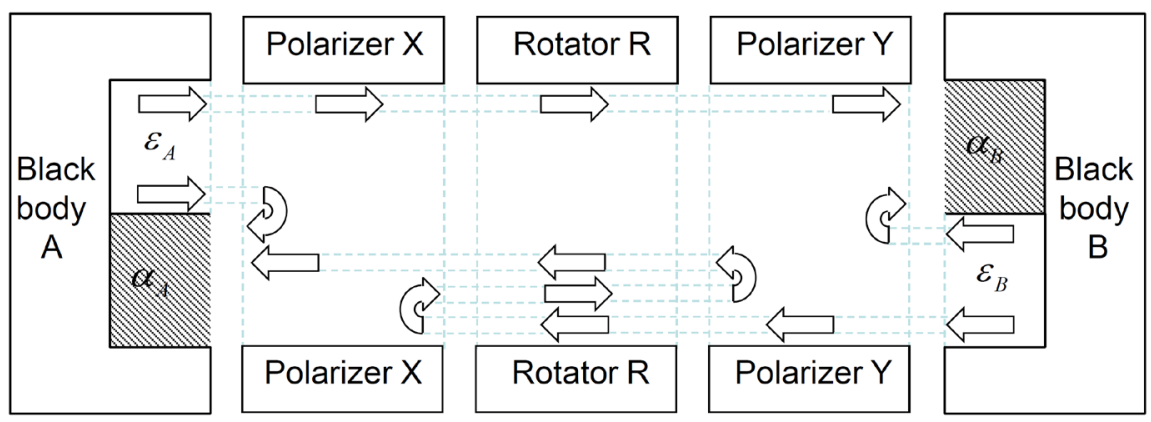

Figure 2. Rayleigh's thought experiment. Black bodies A and B receives the same amount of light. However, the design is faulty as the isolator is not unidirectional. 
eventually re-radiated to the cold object, thereby reestablishing detailed balance.

\section{Analysis of the Faraday Isolator}

The analysis below provides a quantitative analysis of Mungan's argument and finds that Mungan is half right. Light is reradiated backward, but in insufficient amount to prevent a violation of detailed balance. The following thought experiment considers two black bodies A and B separated by a Faraday isolator. The isolator utilizes absorbing polarizers, thereby avoiding the reflection issue raised by the nicols employed by Wien and Rayleigh. The following analysis uses a large number of simple linear equations and is clarified by the drawings.

Before beginning the formal analysis, a baseline shall be established by analyzing a simpler device, that is one in which the polarization rotator within the Faraday isolator is replaced by a black body. Consider the system in Figure 3, comprised of black bodies A and B separated by a polarizer X, a blackbody Z, and a polarizer $\mathrm{Y}$.

In this arrangement, $\mathrm{Z}$ replaces the polarization rotator. The dimensions of the transmission channels are intended to represent how much radiation flows through these channels.

Polarizers $\mathrm{X}$ and $\mathrm{Y}$ are ideal. They transmit half the light and absorb the remaining half. In the figure, emissivity is denoted by $\varepsilon$, absorptivity by $\alpha$ and transmissivity by $\tau$. The system is in thermal equilibrium, therefore, $\varepsilon_{A}=\alpha_{A}$, $\varepsilon_{B}=\alpha_{B}, \quad \varepsilon_{X A}=\alpha_{X B}, \quad \varepsilon_{X Z}=\alpha_{X Z}, \quad \varepsilon_{Y B}=\alpha_{Y B}, \quad \varepsilon_{Y Z}=\alpha_{Y Z}, \quad \varepsilon_{X A}=\alpha_{X B}, \quad \varepsilon_{Z X}=\alpha_{Z X}$, and $\varepsilon_{Z Y}=\alpha_{Z Y}$. Therefore, the black bodies $\mathrm{A}$ and $\mathrm{B}$ are at the same temperature $T_{A}=T_{B}$ as per Stefan-Boltzmann law.

Let us now disturb the equilibrium state by suddenly replacing the black body $\mathrm{Z}$ with an ideal polarization rotator $\mathrm{R}$ as shown in Figure 4. We shall assume initially, as Wien and Rayleigh did, that the rotator is a perfect transmitter, and that it only rotates the plane of polarization without otherwise absorbing or emitting any light. Further down in this paper, we shall show that relaxing this assumption reduces the performance of the device but does not qualitatively change the conclusion of the experiment that detailed balance is violated.

In the instant immediately after the substitution, that is before the objects
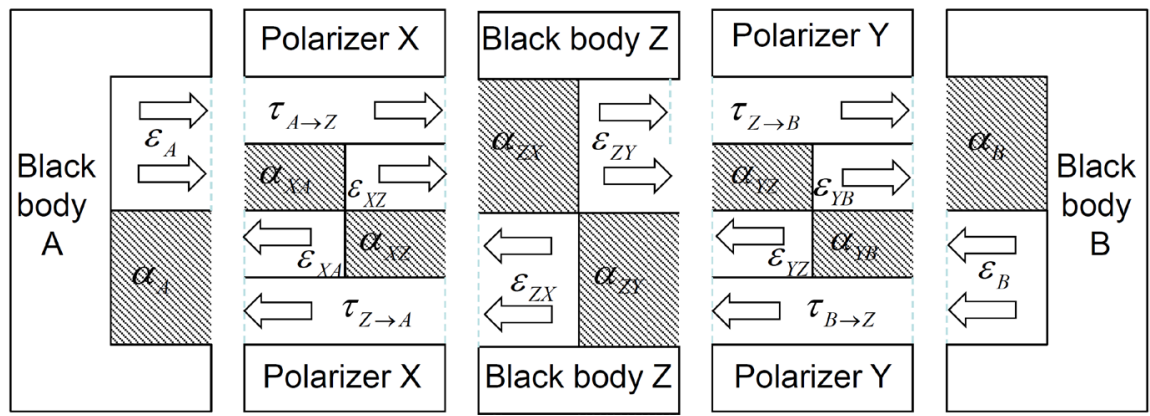

Figure 3. Replacing the polarization rotator $\mathrm{Z}$ of a Faraday isolator by a black body creates a perfectly symmetrical system in which two black bodies A and B reach isothermal equilibrium in compliance with detailed balance. 

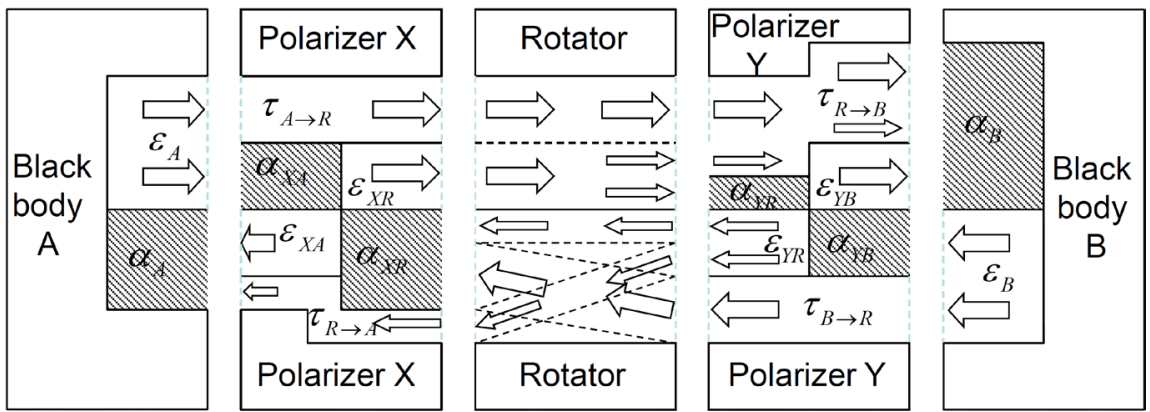

Figure 4. Reinserting the polarization rotator in the Faraday isolator breaks the system's symmetry allowing a temperature difference to develop between two black bodies A and $\mathrm{B}$ on either side of the isolator. Thermal equilibrium is reached when the forward flow of polarized light is compensated by a counter flow of non-polarized light that partially bypasses the input polarizer. The new equilibrium requires a temperature difference between $\mathrm{A}$ and $\mathrm{B}$.

have time to change their temperatures, all emissivities remain the same but absorptivities can change. For example, $\varepsilon_{\mathrm{A}}$ is determined by the temperature of $\mathrm{A}$ before the substitution, therefore $\alpha_{X A}=\varepsilon_{A} / 2$ must also be the same as before. However, $\alpha_{A}=\varepsilon_{X A}+\tau_{R \rightarrow A}$ must change immediately after the substitution because the polarizer transmits less light $\tau_{R \rightarrow A}$. A quick inspection of heat flow shows that $\mathrm{A}$, being upstream in the light flow, absorbs less light immediately after the substitution, indicating that it will get colder after some time elapses. B is downstream, absorbs more light, indicating that it will get warmer. The following analysis solves the large number of linear equations that determine the thermal flow. The reader is invited to rely on the drawing to follow this analysis.

For the sake of simplicity, we define $\varepsilon_{X}=\varepsilon_{X A}=\varepsilon_{X R}$ because $\mathrm{X}$ is at the same temperature throughout. Similarly, $\varepsilon_{Y}=\varepsilon_{Y B}=\varepsilon_{Y R}$. Using these guidelines the following heat flow equations can be inferred by inspection from Figure 3 (before the substitution) and from Figure 4 (after the substitution). Polarizers are assumed to be ideal, allowing through half of non-polarized light, and absorbing the remaining half.

The amount of heat received by A and B immediately after the substitution can be quickly determined.

$$
\alpha_{A}=\varepsilon_{X}+\frac{\varepsilon_{Y}}{2} \quad\left(\text { Note: } \varepsilon_{X}=\varepsilon_{X A}=\varepsilon_{X R} ; \varepsilon_{Y}=\varepsilon_{Y B}=\varepsilon_{Y R}\right)
$$

and

$$
\alpha_{B}=\varepsilon_{Y}+\frac{\varepsilon_{X}}{2}+\frac{\varepsilon_{A}}{2}
$$

Since that the system was in equilibrium just before the substitution $\varepsilon_{X}=\varepsilon_{Y}$ (see Figure 3). Furthermore, since the polarizer is ideal, they transmit half of the light from the black bodies. The polarizers also absorb half of the light from the black bodies and emit half of the light. Hence $\varepsilon_{X}=\varepsilon_{Y}=\varepsilon_{A} / 2$. At the instant immediately following the substitution all temperatures are the same as before the substitution and all emissivities remain the same. Using this information and combining (2) and (3) one can show that 


$$
\frac{\alpha_{B}}{\alpha_{A}}=\frac{5}{3}
$$

which indicates that heat flows from A to B. Interestingly, the polarizers display the opposite tendency with $\mathrm{X}$ absorbing more light and $\mathrm{Y}$ absorbing less light. Since

$$
\alpha_{X A}+\alpha_{X R}=\frac{1}{2} \varepsilon_{A}+\frac{1}{2} \varepsilon_{Y R}+\frac{1}{2} \varepsilon_{B}
$$

and

$$
\alpha_{\mathrm{YB}}+\alpha_{\mathrm{YR}}=\frac{1}{2} \varepsilon_{B}+\frac{1}{2} \varepsilon_{X R}
$$

one can show that

$$
\frac{\alpha_{X A}+\alpha_{X R}}{\alpha_{Y B}+\alpha_{Y R}}=\frac{5}{3}
$$

Immediately following the substitution, heat moves from black body A to black body B, away from the original equilibrium state. One can appreciate that a change does occur. The only way to prevent this shift in equilibrium is for the rotator $\mathrm{R}$ to behave exactly like the black body $\mathrm{Z}$ that it replaces (including being opaque to the polarized light that traverses it), which is obviously impossible even if the elements of the isolator including the polarizers and the rotator were not ideal. Eventually a new equilibrium is reached.

The question is what is the steady state of the black bodies A and B? Assuming zero net heat flow in or out of $\mathrm{A}, \varepsilon_{A}=\alpha_{A}$ :

$$
\varepsilon_{A}=\alpha_{A}=\varepsilon_{X}+\frac{\varepsilon_{Y}}{2} .
$$

Assuming zero net heat flow in or out of $\mathrm{B}, \varepsilon_{\mathrm{B}}=\alpha_{\mathrm{B}}$ :

$$
\varepsilon_{B}=\alpha_{B}=\varepsilon_{Y}+\frac{\varepsilon_{X}}{2}+\frac{\varepsilon_{A}}{2} .
$$

Subtracting Equation (9) from (8) produces

$$
3 \varepsilon_{A}-2 \varepsilon_{B}=\varepsilon_{X}-\varepsilon_{Y} .
$$

Assuming zero net heat flow in or out of $\mathrm{X}, 2 \varepsilon_{X}=\alpha_{X A}+\alpha_{X R}$ :

$$
2 \varepsilon_{X}=\alpha_{X A}+\alpha_{X R}=\frac{\varepsilon_{A}}{2}+\frac{\varepsilon_{Y}}{2}+\frac{\varepsilon_{B}}{2} .
$$

Assuming zero net heat flow in or out of Y, $2 \varepsilon_{Y}=\alpha_{Y B}+\alpha_{Y R}$ :

$$
2 \varepsilon_{Y}=\alpha_{Y B}+\alpha_{Y R}=\frac{\varepsilon_{B}}{2}+\frac{\varepsilon_{X}}{2} .
$$

Subtracting Equation (12) from (11) yields

$$
\frac{\varepsilon_{A}}{5}=\varepsilon_{X}-\varepsilon_{Y} .
$$

Combining with (10) and solving for $\varepsilon_{A}$ and $\varepsilon_{B}$ we find that

$$
\frac{\varepsilon_{B}}{\varepsilon_{A}}=\frac{7}{5}
$$


indicating per Stefan-Boltzmann law that B is hotter than $A, T_{B}>T_{A}$. The polarizers are also at different temperatures. Combining Equations (13) and (8) produces:

$$
\frac{\varepsilon_{X}}{\varepsilon_{Y}}=\frac{11}{8}
$$

If one were to thermally clamp the two polarizers, forcefully setting $T_{X}=T_{Y}$ and $\varepsilon_{X}=\varepsilon_{Y}$, (as one may do in an experimental test of this effect) one would find from Equation (10):

$$
\frac{\varepsilon_{B}}{\varepsilon_{A}}=\frac{3}{2}
$$

This equilibrium state is illustrated in Figure 5 in which the dimensions are approximately proportional to the magnitude of the heat flows. One can see that $\mathrm{B}$ is warmer than A because $\varepsilon_{B}$ is larger than $\varepsilon_{A}$. (This configuration may be more appropriate for an experiment involving film polarizers and rotators in a sandwich).

At room temperature, this difference in emissivity corresponds to a theoretical maximum temperature difference of $32^{\circ} \mathrm{C}$. Assuming polarizers and a rotator, each with a realistic transmissivity of 0.8 , then one can show that $\varepsilon_{B} / \varepsilon_{A}=1.256$ corresponding to a temperature ratio of $T_{B} / T_{A}=1.058$ as per StefanBoltzmann law. At $300 \mathrm{~K}$ the temperature difference is $T_{B}-T_{A}=17.6 \mathrm{C}$ which should easily be observable with careful calorimetric experimental procedures designed to avoid thermal shorts by convection and conduction. For an experiment running at room temperature the challenge is to find polarizers and rotators that operate at the corresponding black body radiation wavelength of about 10 microns.

Let us now revisit the assumption made by Wien and Rayleigh, and that was
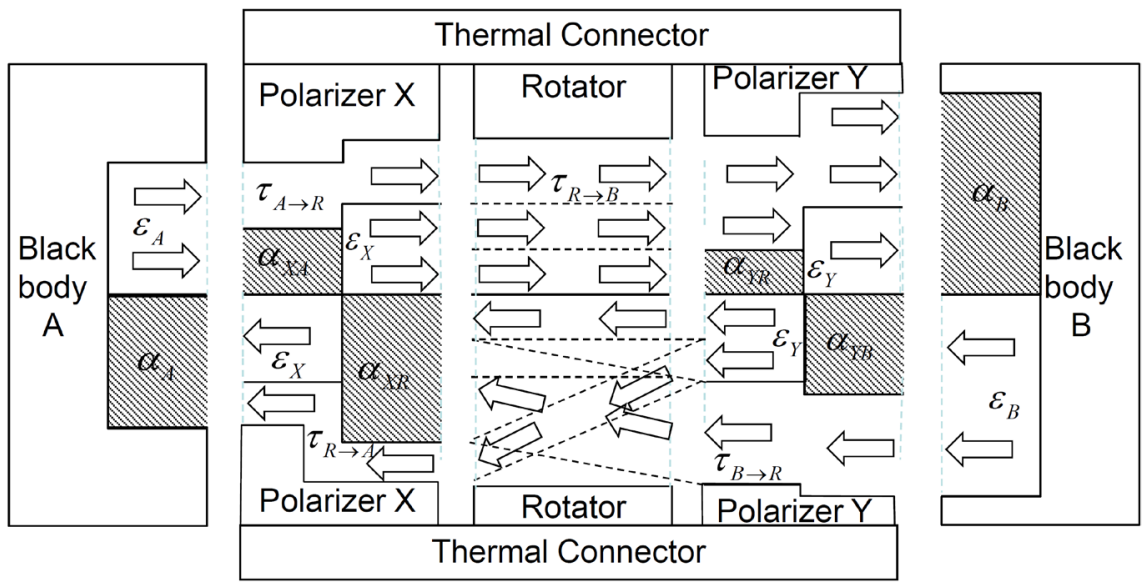

Figure 5. Thermally clamping the polarizers $\mathrm{X}$ and $\mathrm{Y}$ increases the temperature difference between $\mathrm{A}$ and $\mathrm{B}$. The dimensions in the figure are approximately proportional to the conditions of Equation (16). The net flow of heat through the rotator is to the left, indicating that the system is not in static equilibrium and that heat flows to the right through the thermal connector. 
also made in the analysis above. The rotator was assumed to be a perfect transmitter, and that it only rotates the plane of polarization without otherwise absorbing or emitting any light. Let us now consider the case of an imperfect isolator that partially behaves like a black body and partially like a perfect isolator. For example, we could combine Figure 3 and Figure 4 such that the rotator is $50 \%$ comprised of black body and 50\% of perfect rotator. Clearly the performance of the Faraday isolator is degraded but some unidirectionality is retained and a difference in temperature between black bodies A and B still emerges and our conclusion that $T_{B}>T_{A}$ remains qualitatively unchanged.

The thought experiment above began with a modified Faraday isolator in which the rotator is replaced with an internal black body $\mathrm{Z}$ inserted between the two polarizers $\mathrm{X}$ and $\mathrm{Y}$. This arrangement is perfectly symmetrical and isothermal equilibrium is reached. Replacing this internal black body with the rotator $\mathrm{R}$ introduces an asymmetry. Light flows preferentially in one direction causing a temperature difference between the two external black bodies A and B. Eventually a new equilibrium is reached with $T_{B}>T_{A}$, in which the forward flow of light is counterbalanced by a radiative counterflow of internally generated non-polarized light, half of which bypasses the blocking function of the input polarizer. (This explanation is different from Wien who incorrectly relied on a polarized counterflow). Statistical symmetry is restored. Mungan's assertion that the isolator would heat up and cause heat to flow backward thereby avoiding a violation of the second law, is shown to be unsubstantiated by the quantitative analysis above.

Reflective non-reciprocity is also well established for example in optical circulators and ferromagnetic optical materials. The reader is directed to [4] [5] [6] [7] [8] in particular to the non-reciprocal reflective thought experiment by Zhu and Fan [4]. The above discussion applies equally to reflective non-reciprocity.

The above discussion shows that a Faraday isolator placed between two black bodies causes the black body downstream to become hotter than the one upstream. The next question to be addressed is whether the second law and the principle of detailed balance are being violated. Two kinds of systems shall be discussed:

1. Homogeneous Faraday isolator systems.

2. Heterogeneous Faraday isolator systems.

\section{Homogeneous Faraday Isolator Systems}

Can this system convert heat to work, for example by placing a photoelectric device on the colder black body A to capture radiant thermal energy from the hotter black body B? The answer depends on the path taken by photons traveling from $\mathrm{B}$ to the photoelectric device on $\mathrm{A}$.

The Faraday isolator carries thermal energy travels from the colder body A to the warmer body B. This shall be called the forward path. The path taken by photons traveling from $\mathrm{B}$ to the photoelectric device on A shall be called the reverse path. 
If the reverse path is homogenous with the forward path, in other words, if the photons from $B$ go through the Faraday isolator to reach the photoelectric device on $\mathrm{A}$, then, these photons are indistinguishable from those in the forward path and no energy can be generated. This result complies with the $\mathrm{H}$-theorem that requires homogeneity and indistinguishability of particles.

\section{Heterogeneous Faraday Isolator Systems}

If a heat engine is connected between A and B using a reciprocal photon or phonon flow channel, for example, a conventionally conducting material as shown in Figure 6, then useful work can be produced. The reason is that the heat phonons traveling through the thermal connectors are not affected by the non-reciprocity and they can be differentiated from the photons going through the isolator.

A Faraday isolator is a photon diode that produces a temperature difference between its input and output. Using a heterogeneous reverse path, this temperature difference can be used to convert heat to work. How is this concept different from the discredited idea that energy can be produced from the built-in potential in a semiconductor diode by connecting leads across the diode? In a diode, the carriers in the reverse path (the leads) have the same statistics as the ones in the forward path (the junction). The reverse path carriers are subjected to the same potential energy gradients as the diode's carriers. The electrical potentials at the contacts between the diode and the leads cancel out the diode built-in potential.

In contrast, the heat carriers in the system depicted in Figure 6 are different in the forward path and in the reverse path. In the forward path, the heat carriers are photons traveling through the non-reciprocal isolator and in the reverse path, they are phonons in a conventional reciprocal thermal conductor. The two kinds of heat carriers are physically different, have different statistics, and therefore, can be distinguished from each other. The H-theorem does not apply. Heat energy can be extracted from the system.

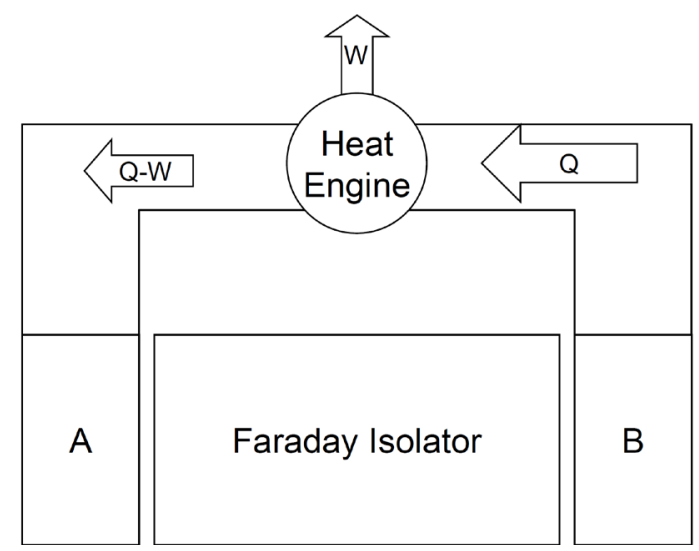

Figure 6. A heat engine can extract useful work from the non-reciprocal transmitter or reflector only if the connections to the heat engine do not go through the non-reciprocity. Such a system is heterogeneous and falls outside the coverage of the H-theorem. 


\section{Conclusions}

This paper explores the limits of applicability of the second law. As stated by the H-Theorem, this law requires that the entropy of an isolated system can never decrease. The theorem, however, makes a crucially important assumption: the system must be homogeneous and its constituent particles indistinguishable. The Faraday isolator is used as a vehicle to exemplify two kinds of systems: the first, in which particles are homogeneously distributed and indistinguishable; the second, in which particles of different species such as fermions and bosons are heterogeneously distributed. This second kind of system falls outside of the H-Theorem.

The paper begins by showing that a temperature difference can arise spontaneously between two black bodies separated by a Faraday isolator.

If a heat engine is thermally connected to these black bodies by particles homogeneous with, and indistinguishable from those in the isolator, (i.e., connecting photons traversing polarizers and rotator similarly configured as in the isolator) then the entire system conforms with the H-Theorem. The connecting photons produce the same temperature difference as those in the isolator. Even though a temperature difference does exist between the black bodies, this temperature difference cannot be communicated to the engine, and no useful work is generated in compliance with the second law.

This phenomenon is reminiscent of the built-in potential across a semiconductor junction. This potential is also unusable. The electrical carriers are homogeneous and indistinguishable throughout the system. Since electrical potential is a scalar field, contact potentials exactly cancel the built-in potential.

Both examples (isolator and semiconductor junction) describe homogeneous systems: the photons in the isolator behave the same as the photons in the thermal connector. Similarly, the electrons in the semiconductor junction have properties indistinguishable from the electrons in the electrical connectors. In such systems, voltage and temperature differences can occur spontaneously but are unusable to produce work. This result complies with the H-Theorem and the second law.

However, heterogeneous systems are not bound by the H-Theorem. As described in this paper, a Faraday isolator can produce a temperature difference between two black bodies. A heterogeneous system can be then formed by connecting a heat engine between the black bodies, using heat carriers with physical properties different from the photons in the isolator. The heat carriers could, for example, be heat phonons in a metallic conductor, not susceptible to the influence of the polarizers and the magnetic field in the rotator. The engine can then produce useful work.

The Faraday isolator is another example uncovered by the author, of heterogeneous systems falling outside the H-Theorem. These systems combine particles with different statistics such as fermions and bosons. In other publications [9] [10] the author shows that under proper conditions, a thermoelectric junction can spontaneously produce a detectable temperature difference even in the 
absence of any electrical input. This effect has been observed in the lab [11] as a failure of the voltage-temperature Seebeck curve to pass through the origin. In the thermoelectric example, a heterogeneous system is formed when the thermal loop is closed by heat phonons in a thermally conductive medium. Since a thermometer can be viewed as a heat engine, the simple act of measuring a temperature difference output that arises without any electrical input constitutes a violation of the second law. Such violations are not mere microscopic fluctuations but have been observed [11] as large scale phenomena and dismissed as unexplained experimental error.

\section{Acknowledgements}

I thank my wife Penny for her unwavering support.

\section{References}

[1] Mungan, C.E. (1999) Faraday Isolators and Kirchhoff's Law: A Puzzle. https://www.usna.edu/Users/physics/mungan/_files/documents/Scholarship/Farada yIsolators.pdf

[2] Wien, W. (1900) Les lois théoriques du rayonnement, Rapports présentés au Congrès International de Physique, Paris, Vol. II, 29.

[3] Rayleigh (1901) On the Magnetic Rotation of Light and the Second Law of Thermodynamics. Nature (London), 64, 577-578. https://doi.org/10.1038/064577e0

[4] Zhu, L. and Fan, S. (2014) Near-Complete Violation of Detailed Balance in Thermal Radiation. Physical Review B, 90, Article Number: 220301. https://doi.org/10.1103/physrevb.90.220301

[5] Krichevtsov, B.B., Pavlov, V.V., Pisarev, R.V. and Gridnev, V.N. (1993) Spontaneous Non-Reciprocal Reflection of Light from Antiferromagnetic $\mathrm{Cr}_{2} \mathrm{O}_{3}$. Journal of Physics. Condensed Matter, 5. https://doi.org/10.1088/0953-8984/5/44/014 http://iopscience.iop.org/article/10.1088/0953-8984/5/44/014/meta

[6] Dumelow, T. and Camley, R.E. (1996) Nonreciprocal Reflection of Infrared Radiation from Structures with Antiferromagnets and Dielectrics. Physical Review B, 54, Article Number: 12232. https://doi.org/10.1103/PhysRevB.54.12232

[7] Stamps, R.L., Johnson, B.L. and Camley, R.E. (1991) Nonreciprocal Reflection from Semi-Infinite Antiferromagnets. Physical Review B, 43, 3626. https://doi.org/10.1103/physrevb.43.3626

[8] He, C., Sun, X., Zhang, Z., Yuan, C., Lu, M., Chen, Y. and Sun, C. (2013) Nonreciprocal Resonant Transmission/Reflection Based on a One-Dimensional Photonic Crystal Adjacent to the Magneto-Optical Metal Film. Optics Express, 21, Article No. 28933. https://doi.org/10.1364/OE.21.028933

[9] Levy, G.S. (2015) Anomalous Temperature Gradient in Non-Maxwellian Gases. In: Ceramics for Energy Conversion, Storage, and Distribution Systems, Proceedings of the 11 th International Conference on Ceramic Materials \& Components for Energy \& Environmental Applications, Vancouver, BC, 14-19 June 2015, Wiley, Hoboken, Vol. 255.

[10] Levy, G.S. (2015) Quantum Game Beats Classical Odds-Thermodynamics Implications. Entropy, 17, 7645-7657. https://doi.org/10.3390/e17117645

[11] Iwanaga, S., Toberer, E.S., LaLonde, A. and Snyder, G.J. (2011) A High Temperature Apparatus for Measurement of the Seebeck Coefficient. Review of Scientific Instruments, 82, 1-6. https://doi.org/10.1063/1.3601358 
Submit or recommend next manuscript to SCIRP and we will provide best service for you:

Accepting pre-submission inquiries through Email, Facebook, LinkedIn, Twitter, etc. A wide selection of journals (inclusive of 9 subjects, more than 200 journals)

Providing 24-hour high-quality service

User-friendly online submission system

Fair and swift peer-review system

Efficient typesetting and proofreading procedure

Display of the result of downloads and visits, as well as the number of cited articles Maximum dissemination of your research work

Submit your manuscript at: http://papersubmission.scirp.org/

Or contact jamp@scirp.org 